\title{
Glucose-6-phosphate dehydrogenase deficiency and hydroxychloroquine in the COVID-19 era: a mini review
}

\author{
Maria Elisabetta Onori ${ }^{1} \cdot$ Claudio Ricciardi Tenore $^{1} \cdot$ Andrea Urbani $^{1,2} \cdot$ Angelo Minucci $^{1}{ }^{1}$
}

Received: 14 October 2020 / Accepted: 12 February 2021 / Published online: 23 February 2021

(c) The Author(s), under exclusive licence to Springer Nature B.V. part of Springer Nature 2021

\begin{abstract}
The coronavirus disease 2019 (COVID-19) is until today a global health emergency. In an immense effort, effective drugs against COVID-19 are searched and intensive researches on possible repurposing of antiviral agents are performed. Since chloroquine (CQ) and hydroxychloroquine (HCQ) have shown in vitro anti- COVID-19 activities, the potential effect of CQ/ HCQ to treat and/or prevent COVID-19 infection has caused global attention. However, concern regarding possible hemolysis in G6PD-deficient COVID-19 patients exists and for this reason, the association between HCQ and G6PD deficiency (G6PDD) is back in the limelight. This study aims to answer the question raised by Mastroianni et al. "Hydroxychloroquine: Culprit or Innocent Bystander in G6PD-Deficient Patients with COVID-19?", reporting all cases of HCQ in G6PD deficient COVID-19 patients published on PubMed (pubmed.ncbi.nlm.nih.gov), in addition to the Mastroianni's patient. In our opinion, after an accurate revision of these cases and responding the question raised by Mastroianni et al., we believe that it is difficult to reach a final verdict about the definitive role of HCQ in these patients. The COVID-19 pandemic has reopened attention on HCQ use and G6PDD. G6PD status is extremely important in modulating the level of reactive oxygen species and many cellular immune responses such as enhanced production of the pro-inflammatory cytokine and inflammasome activation. Since these processes are involved in COVID-19 infection, acute hemolytic anemia, a severe complication of the G6PDD, can occur in these patients. In this context, the role of HCQ, usually effective, safe, and well tolerated in G6PD deficient patients, must be redefined in these patients with COVID-19.As consequence, answering the question: "Hydroxychloroquine: Culprit or Innocent Bystander in G6PD-Deficient Patients with COVID-19?", we state that it is risky to believe that HCQ may be an "innocent bystander" in G6PD-deficient COVID-19 patients.
\end{abstract}

Keywords G6PD deficiency $\cdot$ COVID-19 $\cdot$ Hydroxychloroquine $\cdot$ Hemolytic crisis $\cdot$ Hemoglobin

$\begin{array}{ll}\text { Abbreviations } \\ \text { COVID-19 } & \text { Coronavirus disease } 2019 \\ \text { CQ } & \text { Chloroquine } \\ \text { HCQ } & \text { Hydroxychloroquine } \\ \text { G6PDD } & \text { G6PD deficiency } \\ \text { ICU } & \text { Intensive care unit } \\ \text { Hb } & \text { Hemoglobin } \\ \text { HA } & \text { Hemolytic anemia }\end{array}$

Andrea Urbani

andrea.urbani@policlinicogemelli.it

$\triangle$ Angelo Minucci

angelo.minucci@policlinicogemelli.it

1 Molecular and Genomic Diagnostics Unit, Fondazione Policlinico Universitario A. Gemelli IRCCS, Rome, Italy

2 Catholic University of the Sacred Heart, 00168 Rome, Italy

\section{Introduction}

Chloroquine (CQ) was first synthesized by Hans Andersag in 1934 [1], and it has been used to eradicate malaria and its most devastating agent Plasmodium falciparum for many years. Although CQ produces few adverse effects, high dosage and long-term administration can lead to severe toxicity, including retinopathy, neuropathy, cardiomyopathy, hypoglycemia, dermatological reactions and bone marrow suppression [2]. CQ was not adopted for medical use on a large scale until the early 1940s when hydroxychloroquine (HCQ) was created by replacing an ethyl group in CQ with a hydroxyethyl group; this results in a larger volume of distribution and lower toxicity in humans [3] and its medical use, as an alternative to CQ, was approved in 1955 [4].

The coronavirus disease 2019 (COVID-19) is until today a global health emergency. On January 4th, 2021, COVID19 has led to more than $80,316,555$ confirmed cases and 
1,770,695 deaths worldwide (www.ecdc.europa.eu/). In an immense effort, effective drugs against COVID-19 are searched and intensive researches on possible repurposing of antiviral agents are performed.

$\mathrm{HCQ} / \mathrm{CQ}$ is still a common practice in countries where malaria is endemic, despite drug resistance and WHO recommendations $[5,6]$.

HCQ is also used for the management of autoimmune and rheumatic diseases such as systemic lupus erythematosus [7].The efficacy of HCQ in rheumatic illnesses stems from its anti-inflammatory and immunomodulatory effects. In addition, HCQ has a broad-spectrum antiviral effect against SARS coronavirus, Zika virus, rabies virus, Ebola virus, poliovirus, HIV, influenza $A$ and $B$, hepatitis $A$ and $C$ virus, Chikungunya virus, and Dengue virus. HCO is usually effective, safe, and well tolerated. More frequently the adverse events are related to gastrointestinal complaints, such as abdominal pain, nausea, vomiting and diarrhea.

For these its features and lately since CQ and HCQ have shown in vitro anti- COVID-19 activities [8], the potential effect of CQ/HCQ to treat and/or prevent COVID-19 infection has caused global attention.

With the advent of COVID-19, the association between HCQ and G6PDD is back in the limelight. In fact, a literature review (Sep-2020) on PubMed (pubmed.ncbi.nlm.nih. gov), using "G6PD and COVID-19" as keywords, showed 27 papers of which 17 (62\%) papers with the keywords HCQ, G6PD and COVID-19, and 6 case reports describing G6PDdeficient COVID-19 patients. Therefore, G6PDD is one of the most involved hematological disorders in COVID-19 infection and above all, in patients taking HCQ.

The starting point for our mini review, including all case reports using HCQ in G6PD deficient COVID-19 patients published on PubMed, was stimulated by Mastroianni et al. [9], who raised the question: "Hydroxychloroquine: Culprit or Innocent Bystander in G6PD-Deficient Patient with COVID-19?".

Aware that it may be difficult to definitively explain the role of HCQ in these patients, we aimed to answer the authors' question by reviewing all cases reported in literature.

\section{First case}

The first case is reported by Beauverd et al. [10]. The patient is a 68-year-old Congolese man admitted to the hospital with fever, dyspnea, muscular pain and tiredness due to COVID-19 infection. Amoxicillin/clavulanate was administered on day 1, then changed to piperacillin/tazobactam on day 3. Because of rapid respiratory complications, the patient was transferred to the intensive care unit (ICU), where a single HCQ dose was administered on day 6. During hospitalization, the patient experienced a drop in hemoglobin $(\mathrm{Hb})$ levels from 12 to $6.5 \mathrm{~g} / \mathrm{dL}$, requiring several blood transfusions. Blood results demonstrated biological signs of hemolytic anemia (HA) from day 5. Analysis of a peripheral blood smear demonstrated numerous hemi-ghost cells and microspherocytes not present on day 1. A retrospective review of daily blood smears showed that a significant number of hemi-ghost cells and microspherocytes appeared from day 4 and gradually increased until day 7. Diagnosis of G6PDD was established (Table 1) and a worsening of blood parameters, the day following HCQ administration (day 7), was highlighted.

Based on these evidences, the authors suggested that severe COVID-19 infection triggered HA in this patient. Moreover, the authors stated that HA was possibly worsened by HCQ administration.

\section{Second case}

Maillart et al. [11] described the case of a 65-year-old man, originating from Cameroon, hospitalized for hypoxemia due to COVID-19 infection. Baseline biology showed a $\mathrm{Hb}$ of $13.3 \mathrm{~g} / \mathrm{dl}$. HCQ and azithromycin were started on the admission and on day 2, the patient's condition worsened with $\mathrm{Hb}$ dropped to $11.8 \mathrm{~g} / \mathrm{dl}$, requesting the transfer to the ICU. On day 5, haptoglobin was below detection level, total bilirubin was $4.4 \mathrm{mg} / \mathrm{dl}$, LDH 1743 and HCQ was discontinued. G6PDD was severe (Table 1). The patient, showing $\mathrm{Hb}$ of $7.2 \mathrm{~g} / \mathrm{dl}$, received multiple blood transfusions. The ICU discharge report stated ARDS and HA.

The authors concluded that G6PDD should be considered in case of HA when HCQ is used, particularly in patients of African ascendance.

\section{Third case}

De Franceschi et al. [12] reported a 72-year-old Italian man admitted for fatigue, dyspnea, dizziness and fever. He was suffering of ischemic cardiomyopathy and no episodes of HA were present in the patient's history. Physical examination revealed significantly reduced peripheral saturation in ambient air and the chest X-Ray documented bilateral interstitial pneumonia. Laboratory tests showed normal $\mathrm{Hb}$ levels $(15 \mathrm{~g} / \mathrm{dl})$. The patient was hospitalized and received lopinavir, HCQ and oxygen. After $48 \mathrm{~h}$, an acute drop in $\mathrm{Hb}$ was observed. HCQ was discontinued and the patient received blood transfusions.

De Franceschi et al. concluded that the HA was triggered by HCQ in a patient with suspected G6PDD, then biochemically confirmed [13] (Table 1). 


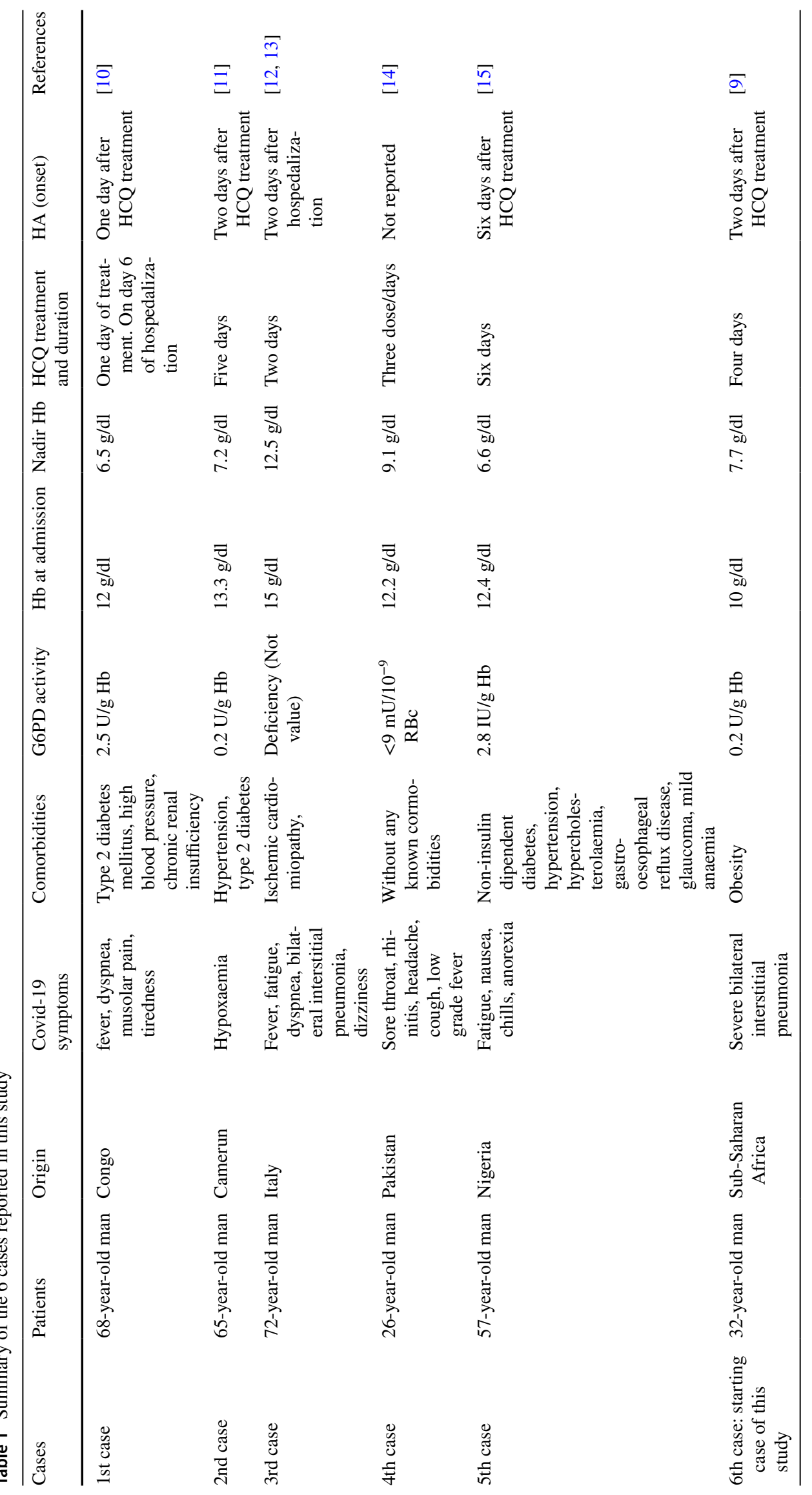




\section{Fourth case}

Sasi et al. [14] reported the case of a 26-year-old Pakistani male, with no known comorbidities and significant family history of diseases or consanguineous marriages. He presented the typical signs of COVID-19 infection. He was treated twice daily with oseltamivir $(150 \mathrm{mg})$, lopinavir/ ritonavir (400 mg/100 mg) and HCQ (400 mg), while once daily with azithromycin $(500 \mathrm{mg})$ and ceftriaxone $(2 \mathrm{~g})$. His $\mathrm{Hb}$ electrophoresis pattern was most consistent with $\mathrm{HbD}$ disease. G6PDD was severe (Table 1) and HCQ was discontinued after 3 doses.

Azithromycin, oseltamivir, and lopinavir/ritonavir were continued at the same dose, while ceftriaxone was changed to piperacillin-tazobactam. His $\mathrm{Hb}$ level dropped from 12.2 to $9.1 \mathrm{~g} / \mathrm{dl}$ over the next 10 days. Hemolysis workup showed normal bilirubin and normal haptoglobin, but elevated LDH. The reticulocyte count was $0.7 \%, 0.8 \%$ and $0.7 \%$ (on admission, on 10 and on 18 days, respectively). Urine dipstick and microscopy were normal and no evidence of hemoglobinuria was demonstrated; $\mathrm{Hb}$ was stable after day 12 and improved to his baseline by day 22 . The patient did not require blood transfusions.

Sasi et al. concluded that this patient did not develop HA, supporting the consideration that SARS-CoV-1, SARSCoV-2, or MERS-CoV do not trigger G6PDD HA. However, the authors recommended the screening of COVID-19 patients for G6PDD, especially before initiation of HCQ therapy.

\section{Fifth case}

Chaney et al. [15] detailed the case of a 57-year-old Nigerian male, who attended the emergency department with a 4 day history of fatigue, nausea, chills and anorexia. The man had a history of non-insulin dependent diabetes, hypertension, hypercholesterolaemia, glaucoma, gastro-oesophageal reflux disease and without known hematology history. He presented a mild anemia on admission with a $\mathrm{Hb}$ of $12.4 \mathrm{~g} /$ dl. Examination revealed dehydration and crepitation at his right lung base, increased work of breathing requiring oxygen. Laboratory results showed a diabetic ketoacidosis. His pneumonia was initially treated with piperacillin/ tazobactam. On day 2, his antibiotic regime was changed to azithromycin, HCQ and ceftriaxone. Laboratory monitoring showed a daily drop in his $\mathrm{Hb}$ reaching $6.6 \mathrm{~g} / \mathrm{dl}$ on day 8 , when the patient was transfused. G6PDD was moderate (Table 1).

Chaney et al. concluded that in this COVID19 patient with increased oxygen requirement, acute severe anemia can complicate the problem and be life threatening. In addition, following the diagnosis of G6PDD, the role of HCQ in the treatment of COVID19 must be carefully evaluated, because it can trigger significant adverse events.

\section{Sixth case}

Mastroianni et al. [9] described the case of a 32-year-old Belgian man, with sub-Saharan African origins, hospitalized with COVID-19 infection. The patient reported no particular clinical history. On admission, he showed a $\mathrm{Hb}$ value of $10 \mathrm{~g} / \mathrm{dl}$. After hospitalization, the patient presented a severe bilateral pneumonia and he started HCQ treatment for 5 days. On day 2 of HCQ administration, a rapid drop of the $\mathrm{Hb}$ level to $7.7 \mathrm{~g} / \mathrm{dl}$ was observed and a complete blood tests showed signs of HA. Severe G6PDD was found (Table 1). Following a single blood transfusion, the patient's condition improved. The patient's $\mathrm{Hb}$ level stabilized to $8.7 \mathrm{~g} / \mathrm{dl}$ after interrupting HCQ.

The authors postulated that, as every viral infection, COVID-19 causes oxidative stress resulting in a HA in G6PD-deficient patients. In particular, the susceptibility to $\mathrm{HA}$, caused by the infection, would increase with the intake of HCQ. Based on these evidences, the authors recommended a careful use of HCQ in G6PD-deficient COVID19 patients.

\section{Concluding remarks}

\section{Hydroxychloroquine: Culprit or innocent bystander in G6PD-deficient patient with COVID-19?}

In light of the 6 cases above reported, we aimed to answer the question case by case.

In the patient of Beauverd et al., with moderate G6PDD, a single dose of HCQ was administered on day 6 . However, the patient showed already signs of hemolysis on day 5 following antibiotic therapy, with a progressive $\mathrm{Hb}$ drop from $12 \mathrm{~g} / \mathrm{dl}$ (day 1) to $6.5 \mathrm{~g} / \mathrm{dl}$ (day 6). Therefore, HCQ would not seem to be the "culprit" but an "accomplice".

In the case of Maillart et al., with severe G6PDD, HCQ administration was started on the admission day. The $\mathrm{Hb}$ dropped from 13.3 to $11.8 \mathrm{~g} / \mathrm{dl}$ at day 2 . In this patients, HCQ would seem to be the "culprit".

In the patient of De Franceschi et al., with G6PDD, HCQ administration was started on the admission and $48 \mathrm{~h}$ after hospitalization an acute drop in $\mathrm{Hb}$ (basal value: $15 \mathrm{~g} / \mathrm{dl}$ ) was observed. HCQ would seem to be the "culprit".

In the patient of Sasi et al., with severe G6PDD, HCQ administration was started on admission and was discontinued after 3 doses. Hb dropped from 12.2 to $9.1 \mathrm{~g} / \mathrm{dl}$ over 10 days. HCQ would seem to be the "accomplice", also in consideration with the coexistence of hemoglobin D disease in this patient. 
In the patient of Chaney et al., with moderate G6PDD, HCQ administration was started $48 \mathrm{~h}$ after admission. $\mathrm{Hb}$ dropped from 12.4 to $6.6 \mathrm{~g} / \mathrm{dl}$ (day 8 ). In this case, HCQ would seem to be the "culprit".

Finally, in the case of Mastroianni et al., with severe G6PDD, HCQ administration was started on the admission and continued for 5 days. On day 2, Hb dropped from 10 (admission day) to $7.7 \mathrm{~g} / \mathrm{dl}$. In this patient, HCQ would seem to be the "culprit".

Firstly, we underline that it is important to pay attention on the $\mathrm{Hb}$ level at admission in COVID-19 patients. In all cases, except for the De Franceschi et al. s' patient, Hb levels at the admission were outside the normal range. It is evident that starting from a lower level and in the presence of HCQ, the critical value of $\mathrm{Hb}$ is reached faster, making these patients suitable for blood transfusions. In this context, it would seem to be that in these 5 G6PDD patients, COVID-19 infection caused a drop in Hb and HCQ acted as an additional trigger. This evidence could have a double explanation.

Several factors can influence the clinical severity in patients with COVID-19. For example, in these patients, age is associated with elevated morbidity and mortality [15]. Aging not only affects the immune response, but also induces a pro-inflammatory state. This indicates that the age associated accumulation of oxidative stress and a reduced anti-oxidative defense can worsen viral infections [17]. G6PD status is extremely important in maintaining redox homeostasis and cellular antioxidant defense by keeping cytotoxic ROS at proper levels. Therefore, we can speculate that G6PDD can complicate the COVID-19 infection, finding fertile ground to trigger HA in these patients, a typical but not frequent complication of the G6PDD. As consequence, in elderly patients with COVID-19, those with G6PDD may become more anemic than those with the normal G6PD activity [18]. We emphasize that 4 of the patients reported were over 55 years old and none with coexisting conditions.

In addition, $\mathrm{Wu}$ et al. [19] found that G6PDD enhanced in vitro cell infection with human coronavirus $229 \mathrm{E}$ ( $\mathrm{HCoV}$ 229E), highlighting that viral gene expression was higher in G6PD deficient fibroblasts and G6PD knockdown cells derived from human lung epithelial cells subjected to viral inoculum compared with control cells. As a result, G6PD deficient cells are more prone to COVID-19 infection and HA could be a clinically severe consequence in these patients.

Secondly, all patients reported are male and unaware of their G6PDD. This finding confirms that G6PD deficient patients are often asymptomatic; therefore, G6PD level evaluation is important in COVID-19 patients from countries where G6PDD is more common, regardless of HCQ administration.
In all 6 cases, G6PDD was not confirmed by molecular testing. We underline that it is always important to characterize the G6PDD at the molecular level to identify the gene alteration in order to establish a correct clinical phenotype/ genotype correlation [20]. G6PD variants are categorized in 5 classes depending on enzymatic activity and the associated clinical presentation [19]. Since none of the patients received a molecular $G 6 P D$ testing, we cannot speculate on the possible contribution of $G 6 P D$ variants on HA. However, we are aware that only class I variants, those with clear symptoms (chronic hemolysis) regardless of a trigger, can cause worse complications for those COVID-19 patients, whose chronic hemolysis can get worse; we underline, however, that in these 6 cases, the presence of a possible class I variant was unlikely.

Thirdly, a consideration must be made on the other factors that may have triggered the HA. Among these factors, we should consider the drugs used in these patients.

Drugs reported in these cases include amoxicillin/clavulanate and piperacillin/tazobactam (first patient), azithromycin (second patient), lopinavir (third patient), oseltamivir, lopinavir/ritonavir, azithromycin, ceftriaxone and piperacillin/tazobactam (fourth patient) and piperacillin/tazobactam, azithromycin and ceftriaxone (fifth case). These drugs could potentially have contributed to the development of HA in these patients, as already reported in literature [22-25]. However, there are no reported cases in which the use of these drugs may have triggered HA in patients with G6PDD.

In addition, while the main target of Covid-19 remains the lung, extra-pulmonary complications are now increasingly reported. Among autoimmune manifestations, autoimmune HA is an unusual finding, recently described in patients with COVID-19 infection [26].

In light of these evidences, we cannot exclude that these 2 factors may have contributed to the clinical picture described in these 6 patients.

\section{Summary}

In the current mini-review, a link between G6PDD, one of the most common enzymopathies, and COVID-19, a massive pandemic, is reported. This link is based on HCQ use and HA in G6PD-deficient patients.

HCQ is still used in countries where malaria is endemic and it is also used for the management of autoimmune and rheumatic diseases such as systemic lupus erythematosus.

G6PDD alters redox homeostasis, affects many cellular immune responses and enhances viral infections. For these reasons, HA, a severe phenotype due to G6PDD, can occur in G6PD-deficient COVID-19 patients. As consequence, HCQ use, usually effective, safe and well tolerated in G6PD deficient patients could be questioned in COVID-19 patients, 
acting as a further trigger in an already altered clinical picture.

Acknowledgements We wish to thank Franziska M. Lohmeyer for reviewing and editing our manuscript.

\section{Compliance with ethical standards}

Conflict of interest The authors declared no potential conflicts of interest.

Ethical approval This study complied with the Ethical Principles for Medical Research Involving Human Subjects according to the World Medical Association Declaration of Helsinki and was certified by the Committee of the Applicable Institution of the Fondazione Policlinico Universitario Agostino Gemelli IRCCS, Rome.

\section{References}

1. Frosch T, Schmitt M, Bringmann G, Kiefer W, Popp J (2007) Structural analysis of the anti-malaria active agent chloroquine under physiological conditions. J Phys Chem B 111:1815-1822. https:// doi.org/10.1021/jp065136j

2. Zhou W, Wang H, Yang Y, Chen ZS, Zou C, Zhang J (2020) Chloroquine against malaria, cancers and viral diseases. Drug Discov Today. https://doi.org/10.1016/j.drudis.2020.09.010

3. Thomé R, Lopes SCP, Costa FTM, Verinaud L (2013) Chloroquine: modes of action of an undervalued drug. Immunol Lett. https://doi. org/10.1016/j.imlet.2013.07.004

4. Browning DJ (2014) Pharmacology of chloroquine and hydroxychloroquine. Hydroxychloroquine and Chloroquine Retinopathy. https:// doi.org/10.1007/978-1-4939-0597-3_2

5. Napoli PE, Nioi M (2020) Global spread of coronavirus disease 2019 and malaria: an epidemiological paradox in the early stage of a pandemic. J Clin Med. https://doi.org/10.3390/jcm9041138

6. Ocan M, Akena D, Nsobya S, Kamya MR, Senono R, Kinengyere AA, Obuku EA (2019) Persistence of chloroquine resistance alleles in malaria endemic countries: a systematic review of burden and risk factors. Malar J. https://doi.org/10.1186/s12936-019-2716-Z

7. Schrezenmeier E, Dörner T (2020) Mechanisms of action of hydroxychloroquine and chloroquine: implications for rheumatology. Nat Rev Rheumatol. https://doi.org/10.1038/s41584-020-0372-x

8. Fan HH, Wang LQ, Liu WL, An XP, Liu ZD, He XQ, Song LH, Tong YG (2020) Repurposing of clinically approved drugs for treatment of coronavirus disease 2019 in a 2019-novel coronavirus-related coronavirus model. Chin Med J (Engl). https://doi. org/10.1097/CM9.0000000000000797

9. Mastroianni F, Colombie V, Claes G, Gilles A, Vandergheynst F, Place S (2020) Hydroxychloroquine in a G6PD-Deficient Patient with COVID-19 Complicated by Haemolytic Anaemia: Culprit or Innocent Bystander? Eur J Case Rep Intern Med. https://doi. org/10.12890/2020_001875

10. Beauverd Y, Adam Y, Assouline B, Samii K (2020) COVID-19 infection and treatment with hydroxychloroquine cause severe haemolysis crisis in a patient with glucose-6-phosphate dehydrogenase deficiency. Eur J Haematol 105:357-359. https://doi.org/10.1111/ ejh. 13432

11. Maillart E, Leemans S, Van Noten H, Vandergraesen T, Mahadeb B, Salaouatchi MT, De Bels D, Clevenbergh P (2020) A case report of serious haemolysis in a glucose-6-phosphate dehydrogenase-deficient COVID-19 patient receiving hydroxychloroquine. Infect Dis (Lond) 52:659-661. https://doi.org/10.1080/23744235.2020.17746 44
12. De Franceschi L, Costa E, Dima F, Morandi M, Olivieri O (2020) Acute hemolysis by hydroxycloroquine was observed in G6PDdeficient patient with severe COVD-19 related lung injury. Eur J Intern Med 77:136-137. https://doi.org/10.1016/j.ejim.2020.04.020

13. De Franceschi L, Costa E, Dima F, Morandi M, Olivieri O (2020) Glucose-6-phosphate dehydrogenase deficiency associated hemolysis in COVID-19 patients treated with hydroxychloroquine/chloroquine: New case reports coming out. Eur J Intern Med 80:103. https ://doi.org/10.1016/j.ejim.2020.08.015

14. Sasi S, Yassin MA, Nair AP, Al Maslamani MS (2020) A Case of COVID-19 in a Patient with Asymptomatic Hemoglobin D Thalassemia and Glucose-6-Phosphate Dehydrogenase Deficiency. Am J Case Rep. https://doi.org/10.12659/AJCR.925788

15. Chaney S, Basirat A, McDermott R, Keenan N, Moloney E (2020) COVID-19 \& Hydroxychloroquine side-effects: Glucose 6-phosphate dehydrogenase deficiency (G6PD) and acute haemolytic anaemia. QJM. https://doi.org/10.1093/qjmed/hcaa267

16. Fauci AS, Lane HC, Redfield RR (2020) Covid-19 - Navigating the Uncharted. N Engl J Med. https://doi.org/10.1056/NEJMe2002387

17. Delgado-Roche L, Mesta F (2020) Oxidative Stress as Key Player in Severe Acute Respiratory Syndrome Coronavirus (SARS-CoV). Infection Arch Med Res 51(5):384-387. https://doi.org/10.1016/j. arcmed.2020.04.019

18. Aydemir D, Ulusu NN (2020) Is glucose-6-phosphate dehydrogenase enzyme deficiency a factor in Coronavirus-19 (COVID-19) infections and deaths? Pathog Glob Health 114(3):109-110. https ://doi.org/10.1080/20477724.2020.1751388

19. Wu YH, Tseng CP, Cheng ML, Tseng CP, Cheng ML, Ho HY, Shih SR, Chiu DTY (2008) Glucose-6-phosphate dehydrogenase deficiency enhances human coronavirus $229 \mathrm{E}$ infection. J Infect Dis 197:812-816. https://doi.org/10.1086/528377

20. Minucci A, Onori ME, Mazzuccato G, Urbani A (2019) Molecular basis of favism triggered by ingestion of frozen pumpkin crosscontaminated with fava beans. Clin Biochem 69:45-47. https://doi. org/10.1016/j.clinbiochem.2019.05.013

21. Minucci A, Moradkhani K, Hwang MJ, Zuppi C, Giardina B, Capoluongo E (2012) Glucose-6-phosphate dehydrogenase (G6PD) mutations database: review of the "old" and update of the new mutations. Blood Cells Mol Dis 48:154-165. https://doi.org/10.1016/j. bcmd.2012.01.001

22. Chan Gomez J, Saleem T, Snyder S, Joseph M, Kanderi T (2020) Drug-Induced Immune Hemolytic Anemia due to AmoxicillinClavulanate: A Case Report and Review. Cureus. https://doi. org/10.7759/cureus.8666

23. Wang Q, He Z, Wu X, Wei Y, Huang J (2020) Hematologic adverse effects induced by piperacillin-tazobactam: a systematic review of case reports. Int J Clin Pharm 42(4):1026-1035. https://doi. org/10.1007/s11096-020-01071-8

24. Lopez C, Kim J, Pandey A, Huang T, DeLoughery TG (2020) Simultaneous onset of COVID-19 and autoimmune haemolytic anaemia. Br J Haematol 190(1):31-32. https://doi.org/10.1111/ bjh. 16786

25. Singh A, Singhania N, Sharma A, Sharma N, Samal S (2020) Ceftriaxone induced immune hemolytic anemia. Cureus. 12(6):e8660. https://doi.org/10.7759/cureus.8660

26. Jawed M, Hart E, Saeed M (2020) Haemolytic anaemia: a consequence of COVID-19. BMJ Case Rep. https://doi.org/10.1136/bcr2020-238118

Publisher's Note Springer Nature remains neutral with regard to jurisdictional claims in published maps and institutional affiliations. 